our series, as in his, water immersion fatalities were limited to the bathtub. In each of our fatal cases the patient had bathed alone in a tub that was almost filled with water. We therefore advise that older epileptics bathe alone only in tubs containing generally no more than $5-7.5 \mathrm{~cm}$ of water. Young children whether epileptic or not, should not be permitted to bathe alone in a bathtub. An alternative method of cleansing consists of showering while seated on the floor of the bathtub, with the drain open so that the water flows out constantly. We prefer that the patient use a handheld showering instrument in the bathtub rather than the overhead, wall-implanted type because the former immediately ceases operation on release of finger pressure.

Contrary to Dr Pearn's recommendation, however, we do not advise that epileptics be allowed to shower alone, at least not in a standing position and especially not in glass or plastic enclosed stalls. Several of our patients who experienced a convulsion while showering fell through glass enclosures and severely injured themselves. In addition, two of our patients who had seizures in a shower stall fell against the tap that regulates the hot water flow and suffered extensive burns. Finally, some of our patients sustained serious bruises and lacerations in association with seizures occurring while showering in the upright position. In environments that include only a wall shower and in cases where the individual insists on bathing in a shower stall we recommend that the patient be seated on an appropriate type of chair or bench during the entire shower.

SAMUEL LivingstoN

LYDIA L PAULI IRVING PRUCE

Samuel Livingston Epilepsy
Diagnostic and Treatment Center,

Baltimore, Maryland

\section{A case of intrinsic asthma}

SIR,-I find it a little difficult to understand why this simple and straightforward case report (23 July, p 250) should have been published under the inappropriate and grandiose title of "Community Clinics in Clinical Pharmacology," but I hope you will permit a practising clinician to voice his disquiet over some of the views expressed in the article.

In the case history reference is made to treatment with "intravenous aminophylline and hydrocortisone," which suggests that this was the only treatment the patient received for his episodes of acute status asthmaticus. I think it should have been made clear in the Advice section that if an attack of asthma is ever sufficiently severe to warrant these forms of intravenous therapy it should always be followed up by a course of oral corticosteroids.

It is stated that the patient's symptoms were due to "intrinsic asthma," but this diagnosis cannot be made without skin sensitivity tests, of which there is no mention in the report.

Since the patient had a peak expiratory flow of only $80 \mathrm{l} / \mathrm{min}$ his asthma must have been quite severe and he therefore ought to have been given an initial course of treatment with an oral corticosteroid preparation to bring his symptoms under control before beginning maintenance treatment with a corticosteroid aerosol. As all respiratory physicians know severe asthma cannot be controlled by a corticosteroid aerosol and it is vital in these circumstances to prescribe an oral corti- costeroid, such as prednisolone, for the first few days.

Although it is true that sodium cromoglycate is seldom effective in elderly asthmatics, it is not correct to say that this drug is of no value in intrinsic asthma, since this is a controversial issue which has not yet been resolved.

I would strongly dispute the final conclusion that the most important point is "to educate the patients in the dangers of overdosing themselves with any inhaled $\beta$ agonist." It is equally important, if not more so, to warn patients that if they fail to obtain the usual degree of relief from the use of a bronchodilator aerosol they are in a potentially dangerous situation and should seek medical help immediately.

IAN W B GRANT

Respiratory Unit,

rn General Hospital,

Edinburgh

SIR,-In the article by Dr G $M$ Bell and Professor M D Rawlins on "A case of intrinsic asthma" (23 July, p 250) Professor Rawlins makes the mistake of dismissing sodium cromoglycate (SCG) as being "of no value for intrinsic asthma." This is at variance with the findings of the MRC collaborative trials reported from London in $1972^{1}$ and jointly from London and Edinburgh in 1976.' Using the $\mathrm{FEV}_{1}$ as an index of improvement the latter paper showed that those patients receiving SCG derived very similar benefit whether their asthma was of extrinsic or intrinsic type. Indeed, none of the patients with intrinsic asthma on placebo continued successfully for the first 52 weeks of the trial, whereas $60^{\circ}$ " of those with the same type of asthma on SCG (with or without isoprenaline) were still on this treatment after 52 weeks with an improved $F E V_{1}$. In view of the benign nature of SCG in terms of side effects (no adrenal suppression or oropharyngeal candidiasis), it is a pity to dismiss its use for intrinsic asthma in the presence of good evidence of the converse.

London SE18

DAVID HoNEYBouRne

1 Brompton Hospital MRC Collaborative Trial, British Medical fournal, 1972, 4, 383.

Northern General Hospital Brompton Hospital MRC 1,361 .

\section{Accident or suicide?}

SIR,-I was very interested to read your leading article on this subject (23 July, p 212) but feel that certain fundamental considerations, if not ignored, have at least been left unmentioned.

In the first place a coroner's verdict is based upon legal rather than medical reasoning and to that extent official suicide rates so far as England and Wales are concerned are quite useless for the purpose of medical statistics.

One can approach the facts in any given case along one or two legal paths. In the first one adopts a presumption against suicide (analogous to the presumption of innocence in a criminal trial-the law never having fully accepted that suicide is not a crime) and, in the absence of any express or implied intention to end life by the deceased, one must conclude that the death was accidental. The second approach does not make any presumption but asks whether $(a)$ in ending his or her own life the deceased must have intended to do so, or (b) whether he or she may have had an expec- tation of merely inflicting serious damage or illness while accepting the possibility of death (especially in self-poisoning) or (c) whether he or she could have suffered the damage entirely inadvertently. In the first case the verdict must be suicide, but in the second and third the evidence of intention falls short of suicide and results in open verdicts. In this approach the verdict of accidental death is reserved for those cases in which there is overwhelming evidence of inadvertence.

A history of mental illness or previous attempts to end life are of minor importance legally in reaching a verdict; indeed, one could argue that it is improper even to consider them since they are analogous to previous convictions in a criminal trial and the verdict in any death must be reached only on the basis of the pathological and circumstantial evidence immediately surrounding the death. Those who are mentally disturbed usually have problems of concentration and it could be argued that they are more accident-prone than those not so disturbed and that the mental condition would therefore equally favour an accidental death in some circumstances. Furthermore, by the rules of chance alone a proportion of depressed persons, alcoholics, drug-dependents, and schizophrenics must be involved in accidents to which they have made only an inadvertent contribution.

Within the near future coroners hope to be freed from some of the rules which at present govern their inquiries. At present when death is unnatural they are bound to hold an inquest, but it is to be hoped that in the future the distinction between the non-inquest and the inquest case may depend on whether or not a second party is directly involved in causing the death. This would be a more useful distinction and it could even be accompanied by a reclassification of deaths in which all self-induced deaths were put into a single category. The present classification of unnatural deaths has to some extent outlived its usefulness from a legal point of view. The responsibility of deciding what is suicide would then fall on the Office of Population Censuses and Surveys, if they still wished to draw a distinction, and their findings would no doubt be more acceptable to the psychiatrists who are interested in this problem.

Herbert H Pilling HM Coroner for South Yorkshire
(West District) Medico-Legal Centre, Sheffield

\section{Induction of labour and perinatal mortality}

SIR,-We would like to continue the discussion started by the paper of Dr Margaret B McNay and others (5 February, p 347), which essentially advocates routine induction of labour as a means of avoiding deaths of "unknown aetiology" among mature babiesthat is, 38 weeks (266 days) or more. Our concern is that the benefits of such a practice in terms of perinatal salvage may be outweighed by the recognised morbidity that accompanies routine induction due to failed induction, an increase in neonatal jaundice, ${ }^{1-\text {. }}$ and hypoxia from excessive oxytocin infusion. ${ }^{6}$ - Unquestionably the occurrence of mature unknown (MU) stillbirths and neonatal deaths represents obstetric failure; but we suggest that techniques other than induction are now available for dealing with this problem. 
We have analysed 122 perinatal deaths which have occurred at St Mary's Hospital, Praed Street, since January 1971 (overall perinatal mortality 19.9 per 1000 ) and find only 16 which were definitely of more than 38 weeks gestational age or weighed more than $2.5 \mathrm{~kg}$. Three deaths were due to severe congenital abnormalities. Eight were attributed to intrapartum hypoxia, of which five were associated with excessive uterine activity in oxytocin-induced labour (one followed rupture of the uterus). We would now regard these eight deaths as preventable by use of current techniques for monitoring fetal heart rate and uterine activity in labour.

Five antepartum deaths remain to be considered. One was due to placental abruption at 284 days and might therefore have been avoided by routine induction. In one intrauterine death was diagnosed exactly at 280 days. Death had clearly occurred some 4-5 days previously and was not therefore preventable by induction at 40 weeks. The remaining three had uncertain dates; in one gestation was still unknown at delivery and the other two were probably 273 and 280 days as estimated by ultrasound.

Even assuming that dating of pregnancies by ultrasound examination at booking will become routine practice in all cases it is difficult to see how routine induction at term would have saved, at best, more than two out of the 16 MU stillbirths.

The crux of the question is whether a policy of routine induction at St Mary's would have been justified. Currently $38^{\circ}$ o of our patients go into labour at 280 days or beyond. This figure must be viewed in conjunction with an induction rate over the past 18 months of $14^{\circ}$ and a perinatal mortality of 10.9 per 1000 (see figure). If the induction policy advocated by $\mathrm{Dr}$ McNay and her colleagues had been employed we estimated that over the 18month period it would have resulted in an additional 300 inductions of labour, which our figures suggest could not have been justified.

Presumably the majority of MU stillbirths which might be prevented by induction are due to failing placental function. We suggest that an alternative approach to the problem is to monitor oestrogen excretion and the antepartum cardiotoxogram, followed by intrapartum fetal monitoring of all patients. This view is supported by the finding that perinatal mortality of babies weighing more than $2.5 \mathrm{~kg}$ has been halved in the past five years. We have concluded that current monitoring techniques, although far from perfect, can be relied on and that we should concentrate our efforts on the preterm (less than 38 weeks' gestation) stillbirths and neonates who now make up almost $70 \%$ of our perinatal mortality.

RICHARD BEARD Philip J STEER

Department of Obstetrics

and Gynaecology,

St Mary's Hospital Medical School,

Davies, D P, et al, British Medical fournal, 1973, 3,

G76. S R, et al, British Medical fournal, 1974, 3, 228. Calder, A A, et al, Lancet, 1974, 2, 1339.

Chalmers, I, et al, British Medical fournal, 1975, 2,

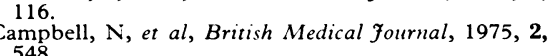

Kubli, F, and Ruttgers, H, in Physiology and Pathology in the Perinatal Period. Leiden, Leiden University Press, 1971 . Liston, W A, and Campbell, A J, British Medical
fournal, 1974, 3, 606 .

\section{Rubella vaccination during oral} contraceptive care

SIR,-Dr Max Gringras and his colleagues (23 July, p 245) show that it is possible to fulfil the recommendation of the Department of Health and Social Security that "any women regularly receiving family planning advice should be screened serologically and if found to be seronegative should be offered rubella vaccination."1

We would comment from our experience ${ }^{2}$ that the implication that such a process, if generally adopted, "would add little to the work load" comes all very well from a group of general practitioners motivated by an interest in research but ignores that littlestudied feature "doctor compliance." Not only this, but laboratory facilities would have to be radically altered to meet a demand for large-scale screening. Our own large district general hospital laboratory co-operated willingly with us because we were engaged in a research project, but normally does only about 20-30 rubella assays per week, of which about one-third are at the request of general practitioners and those largely for obstetric reasons. The DHSS recommendation has evidently, at least in this area, not borne much fruit. We argued that better results are ob- tained by "blind" vaccination of all candidates for contraception irrespective of immunological status and, by offering this at the expiry of a three-month prescription of the pill, the risk of vaccinating the possibly pregnant can reasonably be avoided. Even this policy requires quite an effort of will to implement.

At the same time we appreciate the difficulty facing the DHSS, though no immunisation campaign can be rid of all risk. Admittedly "blind" vaccination throughout the country would sooner or later produce a pregnant vaccinee, though here specific warning to an individual patient is much more malleable a problem than, for instance, general statistics on pertussis vaccination and possible neurological sequelae. It does, however, appear that even then the experts, by distinguishing maternal rubella-specific IgM (the product of recent vaccination) from rubella-specific IgG (produced by a previous rubella infection) could probably advise on the necessity or otherwise of termination and thus solve the problem. The word "probably" in such an emotionally loaded area may not be adequate, and further work on this subject is awaited. The experts furthermore inform us that rubella vaccine, if given to a seropositive woman, has not yet been exonerated from all possible risk.

In spite of these reservations we invite the DHSS to have another think. The fact that vaccination of seronegative women attracts a fee is reasonable under their present policy, but pedantry seems to creep in when payment for vaccinating male staff working in antenatal clinics (not the most likely group to give birth even to normal babies) is withheld unless they have been proved seronegative. Should not family planning clinics, now under area control, have a rubella policy?

It is clear, as both their paper and ours show, that there is still plenty of room for improvement in the campaign against rubella malformations and the fears inspired in so many pregnant women.

A J Rose

KENNETH MOLE

London SW7

Department of Health and Social Security Circular CMO(76)4. London, DHSS, 1976 Rose, A J, and Mole, K F, fournal of the Royal College
of General Practitioners, 1976, 26, 817 .

\section{Late infection after total hip replacement}

SIR,--In your timely reminder of the risks of late infection in total joint replacement following transient bacteraemia (leading article, 23 July, p 213), you neglected to mention what is perhaps the commonest cause of such bacteraemias-dental treatment. It was first shown in $1935^{1}$ that dental manipulations, particularly extractions but also scaling and endodontic therapy, result in transient bacteraemia. It is for this reason that patients with a history of previous rheumatic heart disease, heart murmurs, previous cardiac surgery (valve replacement etc), indwelling pacemaker electrodes, or patent ductus arteriosus should be given prophylactic antibiotic therapy before such treatment.

Rubin et $a l^{2}$ have reported three cases in which a total hip replacement was lost owing to infection after dental treatment, and he presented circumstantial evidence suggesting that it was organisms released into the circulation during dental treatment which were responsible for the infections in the hips. It is important that the medical and dental pro-

Induction rate and perinatal mortality at St Mary's Hospital 